\title{
Asociación entre la participación en programas de asistencia alimentaria y patrones del perfil lipídico en Perú.
}

\section{Association between food assistance program participation and lipid profile patterns in Peru.}

\begin{abstract}
RESUMEN
Evaluamos si la participación en programas de asistencia alimentaria (Comedores Populares y Vaso de Leche) se asocia a patrones de perfil lipídico en población peruana. Análisis secundario de los datos de la Encuesta Nacional de Indicadores Nutricionales, Bioquímicos, Socioeconómicos y Culturales Relacionados con las Enfermedades Crónicas Degenerativas. Se estudió individuos $\geq 20$ años seleccionados de cinco estratos geográficos del Perú. En cada estrato se seleccionó conglomerados por muestreo aleatorio. Se construyeron modelos de regresión de Poisson con varianza robusta para determinar la asociación entre los programas de asistencia alimentaria y el perfil lipídico (colesterol total (CT), HDL-colesterol (HDL-C), LDL-colesterol (LDL-C) y triglicéridos (TG)) de los participantes. De 4028 participantes, solo $123(3,1 \%)$ reportaron ser beneficiarios del programa Comedores Populares y 827 (20,5\%) del Vaso de Leche. En modelo multivariable, se encontró asociación entre ser beneficiario de los Comedores Populares y LDL-c elevado (razón de prevalencia $(R P)=2,33$; IC95\%:1,18-4,59); mientras que ser beneficiario del Vaso de Leche incrementó la probabilidad de tener HDL-c bajo ( $R P=1,08$; IC95\%:1,02$1,14)$, pero redujo la de tener hipertrigliceridemia $(R P=0,70$; IC95\%:0,56-0,88). Ser beneficiario del programa Comedor Popular se asoció a presentar LDL-c elevado; mientras que, ser beneficiario del Vaso de Leche aumentó la probabilidad de presentar HDL-c bajo, pero redujo la probabilidad de presentar hipertrigliceridemia.

Palabras clave: Colesterol; HDL-colesterol; LDL-colesterol; Triglicéridos; Asistencia alimentaria.
\end{abstract}

\footnotetext{
ABSTRACT

Purpose: To assess whether the participation in food assistance programs (Community Kitchens and Glass of Milk) was associated with lipid profile patterns in the Peruvian population. We conducted a secondary data analysis using data from the National Survey of Nutritional, Biochemical, Socioeconomic, and Cultural Indicators related to Chronic Degenerative Diseases. The sample included individuals aged $\geq 20$ years, selected from five geographic strata in Peru. From each stratum a random sample of clusters was chosen.
}

Jacqueline Paredes-Aramburú1, Antonio Bernabé-Ortiz ${ }^{1,2}$.

1. Escuela de Nutrición y Dietética, Facultad de Ciencias de la Salud,
Universidad Peruana de Ciencias Aplicadas, UPC. Lima, Perú.
2. CRONICAS Centro de Excelencia en Enfermedades Crónicas,
Universidad Peruana Cayetano Heredia. Lima, Perú.

Dirigir correspondencia a: Antonio Bernabé-Ortiz. CRONICAS Centro de Excelencia en Enfermedades Crónicas, Universidad Peruana Cayetano Heredia, Lima, Perú. Av. Armendáriz 497, Miraflores, Lima 18, Perú. Teléfono: 511-2416978.

Email: Antonio.Bernabe@upch.pe

Este trabajo fue recibido el 21 de septiembre de 2017. Aceptado con modificaciones: 30 de octubre de 2017. Aceptado para ser publicado: 05 de enero de 2018.

Different Poisson regression models with robust variance were built to determine the association between food assistance programs and participant lipid profile (total cholesterol (TC), $H D L$-cholesterol (HDL-C), LDL-cholesterol and triglycerides (TG)). Data from 4028 participants was analyzed, 123 (3.1\%) reported being beneficiaries of the Community Kitchens program and 827 (20.5\%) were beneficiaries of the Glass of Milk program. An association between being a beneficiary of Community Kitchens and increased LDL-c (Prevalence ratio $(P R)=2.33 ; 95 \% \mathrm{Cl}: 1.18-4.59)$ was found. Being a beneficiary of the Glass of Milk program increased the probability of having low HDL-c levels ( $P R=1.08 ; 95 \% \mathrm{Cl}$ : 1.02-1.14), but reduced the probability of hypertriglyceridemia $(P R=0.70 ; 95 \%$ Cl: 0.56-0.88). Being a beneficiary of the 
Community Kitchen program was associated with increased $L D L$-c levels; while, being a beneficiary of the Glass of Milk increased the probability of low HDL-c, but reduced the probability of developing hypertriglyceridemia.

Key Words: Cholesterol; HDL cholesterol; LDL cholesterol; Triglycerides; Food assistance

\section{INTRODUCCIÓN}

La dislipidemia es considerada un factor de riesgo para la salud cardiovascular. A nivel global, la Organización Mundial de la Salud reportó que la prevalencia de hipercolesterolemia es de $39 \%$ en personas mayores de 25 años de edad. Por ejemplo, la región europea presenta un $54 \%$ de hipercolesterolemia en ambos sexos, mientras que las regiones del sureste de Asia y África reportan cifras de $29 \%$ y $22 \%$, respectivamente 1 . Asimismo, los resultados de una encuesta aplicada en 8 países mostraron que el $9 \%$ de individuos en Tailandia y 53\% en Estados Unidos de América son personas con diagnóstico de hipercolesterolemia ${ }^{2}$. Sin tratamiento, las dislipidemias son un pilar para desarrollar enfermedades crónicas no transmisibles². En el caso del Perú, la prevalencia de hipercolesterolemia se presenta casi en la quinta parte $(19,6 \%)$ de la población peruana mayor de 20 años; mientras que altos niveles de triglicéridos y LDL afectan aproximadamente al $15 \%$ y $13 \%$ respectivamente ${ }^{3,4}$.

En el mundo existen los programas de asistencia alimentarias que cumplen la función de brindar seguridad alimentaria. Por ejemplo, en Canadá tienen la finalidad de asistir a personas de bajos recursos por presentar vulnerabilidad nutricional y sus potenciales consecuencias en la salud ${ }^{5}$. En Estados Unidos de América, estos programas se implementan según la focalización doméstica; mientras que México, utiliza la focalización geográfica y de hogares pero con el mismo fin ${ }^{6}$. En nuestro contexto, según un estudio previo, se determinó que existen 7 millones de beneficiarios de los programas de asistencia alimentaria a nivel nacional ${ }^{7}$. Dos de los programas que tienen cobertura nacional y tienen gran tiempo en Perú son los Comedores Populares y el Vaso de Leche. El Programa de Comedores Populares está organizado por grupos de mujeres voluntarias, comúnmente de un mismo distrito, quienes preparan comida para vender (menús) a precio subsidiado. Esta dirigido a la población en pobreza y pobreza extrema, en riesgo de salud, niños, adultos y personas con discapacidad. Algunos alimentos del menú son subsidiados por el gobierno que entrega alimentos básicos como arroz, trigo, menestras y aceite, mientras que los componentes restantes del plato son adquiridos con las ventas de los menús ocurridas el día anterior ${ }^{8}$. De otro lado, el programa Vaso de Leche está dirigido a niños entre 0 a 13 años, madres gestantes o en periodo de lactancia, sobre todo aquellos en estados de desnutrición. Adicionalmente, el programa puede cubrir individuos de 65 años o más y aquellos afectados por tuberculosis. Este programa es administrado por los gobiernos locales o municipios ${ }^{9}$.

En la actualidad, estos programas tienen el objetivo de mejorar la calidad y seguridad alimentaria. No obstante, trabajos previos en otros países como Estados Unidos de América (EE.UU.) han evidenciado que estos programas (Suplemental Nutrition Assistance Programme, SNAP de sus siglas en inglés) tienen relación con sobrepeso y obesidad $^{10,11}$. Otros estudios, también en USA, han reportado que las mujeres participantes de un programa de asistencia alimentaria tenían mayor probabilidad de tener sobrepeso a diferencia de las que no pertenecían al programa ${ }^{12,13}$. En el Perú se evidenció un panorama similar a los estudios mencionados, en este caso también se encontró que existía un aumento del riesgo de obesidad/sobrepeso en las madres beneficiarias de los Comedores Populares ${ }^{14,15}$. Sin embargo, hasta nuestro conocimiento, no existe evidencia en nuestro contexto sobre el potencial impacto de la participación en los programas de asistencia alimentaria en el perfil lipídico de los usuarios de dichos programas.

Por lo tanto, el objetivo de este estudio fue evaluar si existe asociación entre la participación en programas de asistencia alimentaria (Comedores Populares y Vaso de Leche) y los patrones de perfil lipídico en población peruana.

\section{MATERIALES Y MÉTODOS Tipo de estudio y población de estudio}

Corresponde a un análisis de datos secundarios de una encuesta de base poblacional realizada en Perú. Este análisis secundario utilizó los datos de la "Encuesta Nacional de Indicadores Nutricionales, Bioquímicos, Socioeconómicos y Culturales relacionados con las Enfermedades CrónicoDegenerativas", estudio transversal realizado entre el 2004-2005 y que involucró a personas de 20 años a más que residían en el territorio nacional. Esta encuesta tenía por objetivos caracterizar el estado nutricional y determinar los factores demográficos, socio-económicos y culturales que determinan las características del estado nutricional de la población encuestada.

En el estudio original se incluyeron individuos de 20 años a más de cinco estratos del Perú. Se excluyó a las gestantes o lactantes, personas con malformaciones, y deportistas o físico-culturistas por no permitir una evaluación normal. El muestreo fue multietápico, considerando 5 estratos geográficos: Lima Metropolitana, resto de la Costa, Sierra urbana, Sierra rural y Selva. En cada estrato se seleccionó conglomerados (cuadras o grupos de cuadras) por muestreo simple aleatorio sin reemplazo, y dentro de cada conglomerado, por azar, se seleccionaron viviendas, y de estas los respectivos participantes. Así, de cada estrato se logró enrolar a 840 personas para el estudio.

\section{Población y muestra}

Para este análisis, se incluyeron a todos los encuestados que tenían información completa sobre su participación en programas de asistencia alimentaria y de marcadores bioquímicos en sangre. Basado en un estudio peruano previo ${ }^{3}$ y asumiendo un nivel de confianza del $95 \%$ y un poder del $80 \%$, y que al menos el $4 \%$ de la población pertenece a 
algún programa de asistencia alimentaria y $10 \%$ presenta alguna dislipidemia de interés, se requerían un total de 3,281 participantes para encontrar una asociación con una razón de prevalencia de 2 o mayor.

\section{Definición de variables}

Variable resultado: Cuatro fueron las variables resultado de interés en este estudio, relacionado al perfil lipídico de los participantes basado en colesterol total (CT), HDL-colesterol (HDL-c), LDL-colesterol (LDL-c) y triglicéridos (TG). De esta forma, dislipidemia será definido como: hipercolesterolemia (CT $\geq 200 \mathrm{mg} / \mathrm{dL}$ ), altos niveles de LDL-colesterol (LDL-c $\geq 160$ $\mathrm{mg} / \mathrm{dL}$ ), bajos niveles de HDL-colesterol (HDL-c $<40 \mathrm{mg} / \mathrm{dl}$ en varones $\mathrm{y}<50 \mathrm{mg} / \mathrm{dL}$ en mujeres), e hipertrigliceridemia $(\mathrm{TG} \geq 200 \mathrm{mg} / \mathrm{dL})^{16}$. La toma de muestra se realizó por personal capacitado durante las primeras horas de la mañana y en ayunas. La muestra se obtuvo mediante sistema al vacío con gel activador de la coagulación. Para la obtención del suero la muestra fue centrifugada a $3.000 \mathrm{rpm}$ y almacenada en crioviales. Todas las muestras fueron procesadas por un laboratorio privado. Los niveles de colesterol total, HDL-c y triglicéridos fueron estimados usando el método enzimático Trinder-Colorimétrico ${ }^{17}$, mientras que el LDL-c fue estimado usando la formula de Friedewald ${ }^{18}$.

Exposición: Estuvo basado en el auto-reporte de ser beneficiario de algún programa de asistencia alimentaria (Comedores Populares o Vaso de Leche). Esto fue evaluado mediante la pregunta: " $i$ Es actualmente beneficiario del programa complementario Ilamado Comedor Popular?" o "¿Es actualmente beneficiario del programa complementario llamado Vaso de Leche?". Las opciones de respuesta fueron categorizadas en "Si" o "No". Si bien es cierto que una persona puede ser beneficiaria de ambos programas; usualmente la proporción de estos suele ser baja en adultos.

Otras variables de interés: Se tomaron en cuenta otras variables de interés al evaluar las asociaciones planteadas, entre ellas: sexo (varón vs. mujer), edad $(<40,40-59, y \geq$ 60 años), educación ( $<7,7-11, y \geq 12$ años de educación), nivel socioeconómico (basado en un índice de bienestar creado utilizando posesiones y facilidades de la vivienda, y luego dividido en terciles), región (de acuerdo a los estratos del estudio original), historia de tabaquismo (si/no), nivel de actividad física en el trabajo (alto, medio, y bajo), índice de masa corporal (normal, sobrepeso y obeso, usando los puntos de corte estándar), hipertensión arterial (definido en base a la medida de presión arterial -sistólica $\geq 140 \mathrm{~mm} \mathrm{Hg}$ o diastólica $\geq 90 \mathrm{~mm} \mathrm{Hg}$ - o el antecedente de diagnóstico previo) $^{19}$, y diabetes mellitus tipo 2 (definido en base a una muestra de sangre en ayunas con niveles de glucosa $\geq 126$ $\mathrm{mg} / \mathrm{dL}$ o el antecedente de diagnóstico previo $)^{20}$.

\section{Plan de análisis de datos}

Se usó el paquete estadístico de STATA v.13.0 (Stata Corporation, College Station, TX, USA) para realizar el análisis estadístico correspondiente. Inicialmente se tabuló los datos de la población de estudio en base a las exposiciones (ser beneficiario o no de los programas de asistencia alimentaria en estudio) y a las variables resultado de interés (cada una de las cuatro dislipidemias evaluadas). Se usaron frecuencias y proporciones para las variables categóricas y la asociación entre variables se determinó usando la prueba Chi cuadrado o la prueba exacta de Fisher según correspondiese.

Luego, se calcularon las prevalencias de cada una de las dislipidemias de interés y sus respectivos intervalos de confianza al 95\% (IC95\%). Para hallar la asociación entre las variables de exposición y de respuesta se utilizó los modelos de regresión de Poisson con varianza robusta ${ }^{21}$, reportándose razones de prevalencia (RP) y sus respectivos IC95\%.

\section{Aspectos éticos}

El protocolo de investigación fue revisado y aprobado por el Comité de Ética de la Universidad Peruana de Ciencias Aplicadas (UPC). Esta investigación se ajusta a los principios de la Declaración de Helsinki y a la legislación del Perú. La base de datos usada es de acceso público, y solo cuenta con códigos en vez de identificadores personales para garantizar el anonimato y la confidencialidad respectiva.

\section{RESULTADOS}

\section{Características de la población de estudio}

De un total de 4.209 sujetos enrolados en el estudio original, los datos de 4.028 fueron analizados para el presente estudio, 2.012 (50.1\%) mujeres, y un promedio de edad de $42,1 \pm 15,3$. Del total evaluado, $20.1 \%$ pertenecían a Lima Metropolitana, mientras que $18,9 \%$ eran del resto de la Costa. Por otro lado, solo 123 (3,1 \%, IC95\%: 2,5-3,6) reportaron ser beneficiarios del programa Comedores Populares, mientras que 827 (20,5\%; IC95\%: 19,3-21,8) reportaron ser beneficiarios del Vaso de Leche. Solo 49 (1,2\%) del total de participantes reportaron estar en ambos programas de asistencia alimentaria. Las características de la población de estudio de acuerdo a si eran o no beneficiarios de los programas de asistencia alimentaria evaluados se detallan en la Tabla 1.

\section{Perfil lipídico de los participantes}

Un total de 699 (17,4\%; IC95\%: 16,2-18,5) sujetos presentaron hiper-colesterolemia, mientras que 149 (3,7\%; IC95\%: 3,1-4,3) tuvieron niveles altos de LDL-c, y 599 (14,9\%; IC95\%: 13,8-16,0) presentaron altos niveles de triglicéridos en sangre. De otro lado, 2215 (55,0\%; IC95\%: $53,5-56,5)$ de los individuos evaluados presentaron bajos niveles de HDL-c. Las Tablas 2 y 3 presentan los detalles de la distribución de variables de acuerdo al perfil lipídico de los participantes.

\section{Programas de Asistencia Alimentaria y Perfil Lipídico}

En modelo multivariable, y ajustando por sexo, edad, nivel educativo, nivel socioeconómico, región, tabaquismo actual, niveles de actividad física en el trabajo, índice de masa corporal, antecedente de hipertensión y diabetes mellitus, se encontró asociación entre ser beneficiario de los Comedores Populares y LDL-c elevado $(\mathrm{RP}=2,33$; 


\section{Tabla 1.}

Descripción de la población de estudio según Programas de Asistencia Alimentaria.

\begin{tabular}{cccccc}
\multicolumn{2}{c}{ Comedores Populares } & \multicolumn{2}{c}{ Vaso de Leche } \\
No & $\mathrm{Si}$ & $\mathrm{p}$ & No & $\mathrm{Si}$ \\
$(\mathrm{n}=\mathbf{3 , 9 0 5 )}$ & $(\mathrm{n}=\mathbf{1 2 3})$ & & $(\mathrm{n}=3201)$ & $(\mathrm{n}=\mathbf{8 2 7})$
\end{tabular}

\section{Sexo}

Mujer

$1.947(49,9 \%) \quad 65(52,9 \%) \quad 0,51$

$1.560(48,7 \%)$

$452(54,7 \%)$

0,002

\section{Edad}

$<40$ años

40-59 años

$60+$ años

Nivel educativo*

$\begin{array}{lccc}<7 \text { años } & 1.489(38,1 \%) & 72(58,5 \%) & <0,001 \\ 7-11 \text { años } & 1.403(35,9 \%) & 43(35,0 \%) & \\ 12+\text { años } & 1.012(25,9 \%) & 8(6,5 \%) & \end{array}$

Nivel socioeconómico

Bajo

Medio

Alto

\section{Región}

Lima Metropolitana

Resto de Costa

Selva

Sierra Rural

Sierra Urbana

$\begin{array}{lll}1.326(34,0 \%) & 69(56,1 \%) & <0,001 \\ 1.418(36,3 \%) & 39(31,7 \%) & \\ 1.161(29,7 \%) & 15(12,2 \%) & \end{array}$

$\begin{array}{ccc}781(20,0 \%) & 30(24,4 \%) & <0,001 \\ 743(19,0 \%) & 18(14,6 \%) & \\ 789(20,2 \%) & 17(13,8 \%) & \\ 758(19,4 \%) & 51(41,5 \%) & \\ 834(21,4 \%) & 7(5,7 \%) & \end{array}$

\section{Tabaquismo actual*}

$$
\mathrm{Si}
$$

\section{Actividad física*}

Alto

Medio

Bajo

\section{Índice de masa corporal*}

Normal

Sobrepeso

Obeso

$\begin{array}{ccc}1.928(49,4 \%) & 60(48,8 \%) & 0,71 \\ 1.380(35,3 \%) & 47(38,2 \%) & \\ 597(15,3 \%) & 16(13,0 \%) & \end{array}$

$1.012(25,9 \%) \quad 8(6,5 \%)$
$669(17,2 \%) \quad 27(22,0 \%) \quad 0,17$

0,68

$$
\begin{array}{ccc}
1.503(47,0 \%) & 485(58,7 \%) & <0,001 \\
1.194(37,3 \%) & 233(28,2 \%) & \\
504(15,7 \%) & 109(13,2 \%) &
\end{array}
$$

$$
\begin{array}{ccc}
1.138(35,5 \%) & 423(51,2 \%) & <0,001 \\
1.151(36,0 \%) & 295(35,7 \%) & \\
912(28,5 \%) & 108(13,1 \%) &
\end{array}
$$

$$
\begin{array}{ccc}
959(30,0 \%) & 436(52,7 \%) & <0,001 \\
1.178(36,8 \%) & 279(33,7 \%) & \\
1.064(33,2 \%) & 112(13,5 \%) &
\end{array}
$$

$\begin{array}{ll}673(21,0 \%) & 138(16,7 \%) \\ 653(20,4 \%) & 108(13,1 \%) \\ 666(20,8 \%) & 140(16,9 \%) \\ 522(16,3 \%) & 287(34,7 \%) \\ 687(21,5 \%) & 154(18,6 \%)\end{array}$

$<0,001$

$571(17,9 \%)$

$125(15,2 \%)$

0,06

$\begin{array}{cll}806(25,2 \%) & 303(36,6 \%) & <0,001 \\ 1.333(41,7 \%) & 295(35,7 \%) & \\ 1.061(33,2 \%) & 229(27,7 \%) & \end{array}$

$1.707(53,6 \%)$

$514(62,7 \%) \quad<0,001$

$1.050(33,0 \%)$

$224(27,3 \%)$

$427(13,4 \%)$

$82(10,0 \%)$

$604(19,1 \%)$

$126(15,5 \%)$

0,02

$709(18,4 \%) \quad 21(17,1 \%) \quad 0,70$

0,39

$123(3,9 \%)$

$16(2,0 \%)$

0,008

*Columnas pueden no sumar debido a valores faltantes. 
Tabla 2.

Descripción de la población de acuerdo a Hipercolesterolemia y LDL-c elevado.

\begin{tabular}{|c|c|c|c|c|}
\hline & \multicolumn{2}{|c|}{ Hipercolesterolemia } & \multicolumn{2}{|c|}{ LDL-c elevado } \\
\hline & $\mathrm{n} / \mathrm{N}(\%)$ & $\mathbf{p}$ & $\mathrm{n} / \mathrm{N}(\%)$ & $\mathbf{p}$ \\
\hline \multicolumn{5}{|l|}{ Sexo } \\
\hline Mujer & $304 / 2.016(15,1 \%)$ & & $53 / 2.016(2,6 \%)$ & \\
\hline \multicolumn{5}{|l|}{ Edad } \\
\hline 40-59 años & $348 / 1.427(24,4 \%)$ & & $74 / 1.427(5,2 \%)$ & \\
\hline $60+$ años & $169 / 613(27,6 \%)$ & & 44/613 (7,2\%) & \\
\hline \multicolumn{5}{|l|}{ Nivel educativo } \\
\hline$<7$ años & $260 / 1.561(16,7 \%)$ & 0,23 & $53 / 1.561(3,4 \%)$ & 0,14 \\
\hline $7-11$ años & $244 / 1.446(16,9 \%)$ & & 48/1.446 (3,3\%) & \\
\hline Medio & $252 / 1.457(17,3 \%)$ & & $52 / 1.457(3,6 \%)$ & \\
\hline Alto & $272 / 1.176(23,1 \%)$ & & $61 / 1.176(5,2 \%)$ & \\
\hline \multicolumn{5}{|l|}{ Región } \\
\hline Lima Metropolitana & $167 / 811(20,6 \%)$ & $<0,001$ & $38 / 811(4,7 \%)$ & $<0,001$ \\
\hline Resto de Costa & $182 / 761(23,9 \%)$ & & $45 / 761(5,9 \%)$ & \\
\hline Selva & $92 / 806(11,4 \%)$ & & $19 / 806(2,4 \%)$ & \\
\hline Sierra Rural & $99 / 809(12,2 \%)$ & & $17 / 809(2,1 \%)$ & \\
\hline Sierra Urbana & $159 / 841(18,9 \%)$ & & $30 / 841(3,6 \%)$ & \\
\hline \multicolumn{5}{|l|}{ Tabaquismo actual } \\
\hline No & $581 / 3.316(17,5 \%)$ & 0,65 & $122 / 3.316(3,7 \%)$ & 0,94 \\
\hline \multicolumn{5}{|l|}{ Actividad física (trabajo) } \\
\hline Bajo & $269 / 1.290(20,9 \%)$ & & $65 / 1.290(5,0 \%)$ & \\
\hline \multicolumn{5}{|l|}{ Índice de masa corporal } \\
\hline Normal & $239 / 2.221(10,8 \%)$ & $<0,001$ & $50 / 2.221(2,3 \%)$ & $<0,001$ \\
\hline Sobrepeso & $278 / 1.274(21,8 \%)$ & & $69 / 1.274(5,4 \%)$ & \\
\hline Obeso & $180 / 509(35,4 \%)$ & & $30 / 509(5,9 \%)$ & \\
\hline \multicolumn{5}{|l|}{ Hipertensión } \\
\hline No & $471 / 3.241(14,5 \%)$ & $<0,001$ & 95/3.241 (2,9\%) & $<0,001$ \\
\hline $\mathrm{Si}$ & $216 / 730(29,6 \%)$ & & $52 / 730(7,1 \%)$ & \\
\hline \multicolumn{5}{|l|}{ Diabetes mellitus } \\
\hline No & $653 / 3.865(16,9 \%)$ & $<0,001$ & $138 / 3.865(3,6 \%)$ & 0,008 \\
\hline $\mathrm{Si}$ & 43/139 (30,9\%) & & $11 / 139(7,9 \%)$ & \\
\hline \multicolumn{5}{|l|}{ Comedores Populares } \\
\hline No & $678 / 3.905(17,4 \%)$ & 0,93 & $141 / 3.905(3,6 \%)$ & 0,09 \\
\hline $\mathrm{Si}$ & $21 / 123(17,1 \%)$ & & 8/123 (6,5\%) & \\
\hline \multicolumn{5}{|l|}{ Vaso de Leche } \\
\hline No & $597 / 3.201(18,7 \%)$ & $<0,001$ & $127 / 3.201(4,0 \%)$ & 0,08 \\
\hline $\mathrm{Si}$ & $102 / 827(12,3 \%)$ & & $22 / 827(2,7 \%)$ & \\
\hline
\end{tabular}


Tabla 3.

Descripción de la población de acuerdo a Hipertrigliceridemia y HDL-c bajo.

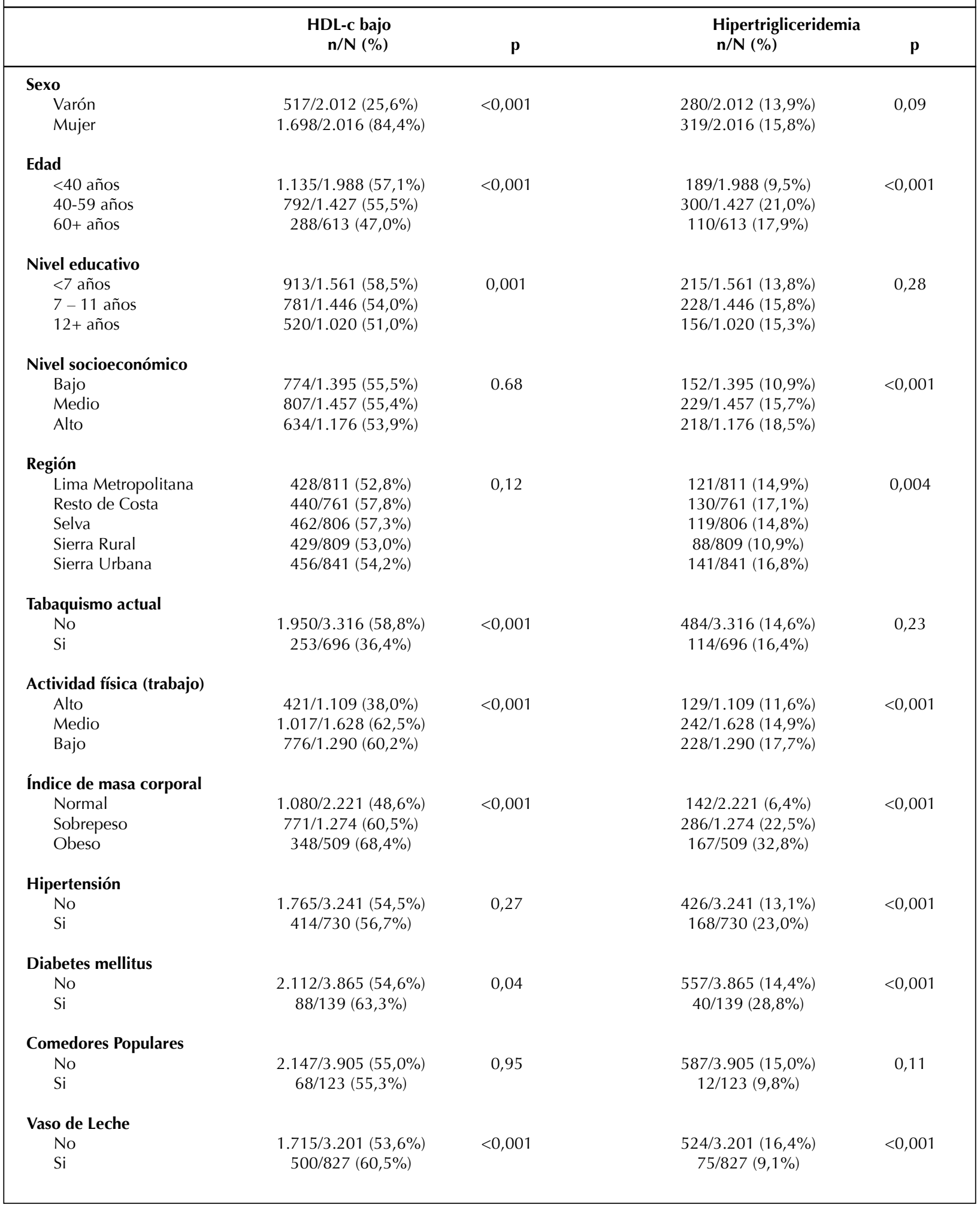


IC95\%: 1,18-4,59). De otro lado, ser beneficiario del Vaso de Leche incrementó la probabilidad de tener HDL-c bajo $(\mathrm{RP}=1,08$; IC95\%: 1,02-1,14), pero redujo la probabilidad de tener hipertrigliceridemia ( $\mathrm{RP}=0,70$; IC95\%: 0,56-0,88). Ver resultados en Tabla 4.

\section{DISCUSIÓN \\ Hallazgos principales}

Nuestros hallazgos evidencian asociación entre ser beneficiario de un programa de asistencia alimentario y ciertos patrones de dislipidemias. Así, ser beneficiario del programa Comedor Popular estuvo asociado a más del doble de probabilidad de presentar LDL-c elevado. Ser beneficiario del Vaso de Leche, sin embargo, tuvo resultados contradictorios: aumentó la probabilidad de presentar HDL-c bajo, pero redujo la probabilidad de presentar hipertrigliceridemia. De otro lado, este estudio confirma que la dislipidemia más frecuente en el contexto peruano es HDL-c bajo, presente en más del $50 \%$ de la población peruana por encima de 20 años de edad.

\section{Comparación con otros estudios}

Existe limitada evidencia del potencial impacto de los programas de asistencia alimentaria sobre el perfil lipídico de sus beneficiarios. Nuestros resultados corroboran un estudio transversal realizado en USA que evaluó la participación en el SNAP y su potencial asociación con diferentes factores metabólicos: ser beneficiario del SNAP estuvo asociado a hipertrigliceridemia, niveles bajos de HDL-c, así como niveles de glucosa elevada y síndrome metabólico ${ }^{22}$. Más aun, esta asociación persistió después de controlar los modelos por el índice de masa corporal. Nuestros hallazgos sin embargo, expanden los resultados del estudio previo mostrando la asociación de dos programas de asistencia alimentaria con diferentes marcadores del perfil lipídico: LDL-c elevado en usuarios de los Comedores Populares y menos hipertrigliceridemia en los del Vaso de Leche.

Otros estudios han mostrado asociación longitudinal y transversal entre ser beneficiario de los programas de asistencia alimentaria y obesidad. Por ejemplo, un estudio longitudinal en Perú encontró que las madres beneficiarias de los Comedores Populares tenían el doble de riesgo de desarrollar obesidad ${ }^{14}$. En México, los programas asistenciales condicionales de dinero han sido asociados a mayor IMC, mayores niveles de presión arterial y mayor sobrepeso ${ }^{23}$. Más aun, en Chile existe evidencia de la asociación entre obesidad en niños y su participación en dos programas de asistencia alimentaria ${ }^{24}$.

De acuerdo a estudios previos, la asociación encontrada podría ser debida a la baja calidad de la dieta consumida por los beneficiarios, especialmente en el caso de Comedores Populares (ej. bajo consumo de frutas, verduras y granos) comparados con los que no son beneficiarios ${ }^{25,26}$. Existe asimismo, evidencia de estudios transversales que no muestran asociación con obesidad o incremento del IMC a pesar de usar metodologías similares a la de nuestro estudio ${ }^{27-29}$.

Tabla 4.

Asociación entre ser beneficiario de los Programas de Asistencia Alimentaria y el perfil lipídico: modelos crudos y ajustados.

Beneficiario de:

\begin{tabular}{cc}
\hline $\begin{array}{c}\text { Comedores Populares } \\
\text { Si (vs. No) }\end{array}$ & Vaso de Leche \\
Si (vs. No)
\end{tabular}

$\begin{array}{llrr}\text { Hipercolesterolemia } & \begin{array}{l}\text { Crudo } \\ \text { Ajustado* }\end{array} & 0,98(0,66-1,46) & 0,66(0,54-0,80) \\ & & 1,16(0,80-1,68) & 0,86(0,71-1,05) \\ \text { LDL-c elevado } & \text { Crudo } & 1,80(0,90-3,59) & 0,67(0,43-1,05) \\ & \text { Ajustado* } & 2,33(1,18-4,59) & 0,96(0,60-1,56) \\ \text { HDL-c bajo } & & 1,00(0,86-1,18) & 1,13(1,06-1,20) \\ & \text { Crudo } & 0,97(0,86-1,10) & 1,08(1,02-1,14) \\ \text { Hipertrigliceridemia } & \text { Ajustado* } & & 0,55(0,44-0,70) \\ & \text { Crudo } & 0,65(0,38-1,12) & 0,70(0,56-0,88)\end{array}$

*Ajustado por sexo, edad, nivel educativo, nivel socioeconómico, región, tabaquismo actual, niveles de actividad física en el trabajo, índice de masa corporal, antecedente de hipertensión, y de diabetes. 


\section{Relevancia en salud pública}

Para el campo nutricional de la evaluación, prevención y control de las enfermedades crónicas no transmisibles, estos hallazgos constituyen un reto importante. Usualmente, los programas de asistencia alimentaria tienen el objetivo de mejorar la salud nutricional de sus beneficiarios y están dirigidos a personas de escasos recursos, y por ende, en riesgo de salud. En ese sentido, el presente trabajo deja como precedente el posible impacto que puede tener ser beneficiario de algunos de los programas de asistencia alimentaria sobre el perfil lipídico, asociándolo a elevación del LDL-c y disminución del HDL-c.

El Vaso de Leche tiene una ración de alimentos ya definida basado en los valores nutricionales mínimos de macro y micronutrientes ${ }^{30}$. El componente mayoritario de la ración son insumos como harina de quinua, kiwicha, haba, maca, cebada, avena, arroz y soya, incluidos por ser alimentos de alto valor nutricional y con menor costo (aproximadamente $207 \mathrm{Kcal}$ con 12\%-15\% de proteínas y 20\%-25\% de grasas). La Organización Mundial de la Salud recomienda que una alimentación sana incluya frutas y verduras, pero también legumbres y cereales integrales, estos últimos brindado por el Vaso de Leche, elementales para prevenir las dislipidemias ${ }^{31}$. Así, la dieta provista por este programa podría ser más saludable y por lo tanto, reduciría la probabilidad de hipertrigliceridemia. En el caso de los Comedores Populares, los menús contienen dos platos (entrada, generalmente una sopa, y un segundo) centrados principalmente en carbohidratos incluyendo tubérculos y arroz, pero también menestras ${ }^{32}$. El menú no contiene frutas o verduras, y en un estudio previo se evidenció que solo el $10 \%$ de los beneficiarios consumía la cantidad de frutas y verduras recomendadas ${ }^{33}$. El exceso de carbohidratos provisto por los menús y la ausencia de verduras y frutas podrían favorecer la elevación del LDL-c.

Los programas de asistencia alimentaria, especialmente los Comedores Populares elaboran, venden (a precio subsidiado), y sirven comida preparada, y podrían ser usados para introducir intervenciones para mejorar la calidad de la dieta de los beneficiarios. Por tal razón, debería haber una mejora del contenido nutricional que dichos programas ponen al alcance de los más necesitados ${ }^{34}$, incluyendo la capacitación y entrenamiento del personal encargado de los programas, así como la inclusión de estrategias promocionales. Nuestros hallazgos señalan la necesidad de implementar estrategias apropiadas para reducir la aparición de condiciones crónicas.

\section{Fortalezas y Limitaciones}

La principal fortaleza del estudio radica en la muestra recolectada, ya que es representativa de la población del Perú. Todas las medidas incluidas en el análisis fueron recolectadas por personal capacitado en lugar de autoreportes como estudios previos, con lo que se disminuyó el potencial de clasificación errónea de nuestras variables de interés.
Se debe destacar, asimismo algunas limitaciones del presente trabajo. Primero, la naturaleza transversal del estudio no permite determinar causalidad, sino solo asociación. Segundo, la variable de exposición (ser beneficiario de un programa de asistencia alimentaria) no fue cuantificada de la mejor manera, dejando de lado la frecuencia de uso del programa, y asumiendo que si usa el programa, este uso es de largo plazo, lo que podría generar mala clasificación. En ese sentido, nuevos estudios, especialmente de naturaleza prospectiva, deberían ser realizados para corroborar nuestros hallazgos. Finalmente, algunos potenciales confusores como los patrones de dieta o los niveles de actividad física, especialmente asociados con dislipidemias ${ }^{31,32}$, no fueron considerados en los modelos de regresión final porque no estaban disponibles.

\section{CONCLUSIONES}

Ser beneficiario del programa Comedor Popular estuvo asociado a más del doble de probabilidad de presentar LDL-c elevado. De otro lado, ser beneficiario del Vaso de Leche, sin embargo, tuvo resultados contradictorios: aumentó la probabilidad de presentar HDL-c bajo, pero redujo la probabilidad de presentar hipertrigliceridemia. Nuestros hallazgos sugieren la necesidad de intervenciones para mejorar la dieta provista en ciertos programas de asistencia alimentaria.

Conflictos de interés: Los autores declaran no tener conflictos de interés.

\section{BIBLIOGRAFIA}

1. Global Health Observatory (GHO) data: Raised cholesterol. WHO, 2017. (Accessed June 14, 2017, at http://www.who. int/gho/ncd/risk_factors/cholesterol_text/en/.)

2. Roth GA, Fihn SD, Mokdad AH, Aekplakorn W, Hasegawa T, Lim SS. High total serum cholesterol, medication coverage and therapeutic control: an analysis of national health examination survey data from eight countries. Bull World Health Organ 2011; 89: 92-101.

3. Cardenas H. National Survey of Nutritional and Biochemical Indicators for Noncommunicable Diseases. Lima, Peru: Instituto Nacional de Salud: Centro Nacional de Alimentación y Nutrición; 2006.

4. Panamerican Health Organization. Health in the Americas: Peru. Washington DC, US: PAHO; 2012.

5. Gaetz S, O'Grady B, Buccieri K, Karabanow J, Marsolais A. Youth homelessness in Canada: Implications for Policy and Practice. Toronto, Canada: Canadian Homelessness Research Network Press; 2013.

6. Gundersen MY, Valdez C, Kuhn B. A comparison of food assistance program in Mexico and the United States. Washington DC, US: Economic Research Service/USDA; 2000.

7. Programa Mundial de Alimentos. Proposal of Reforms of Food and Nutrition Programs in Peru. Lima, Peru: PMA; 2010.

8. Kamioka N. Women's voluntary groups in Lima, Peru Comedores Populares. Washington DC, US: Latin American Association; 2001.

9. Ministerio de Economia y Finanzas. Glass of Milk Program. 2017. (Accessed Junio 10, 2017, at https://www.mef.gob.pe/ 
es/transferencias-a-gobierno-nacional-regional-y-locales/baselegal-y-aspectos-metodologicos/programa-vaso-de-leche.)

10. Kimbro RT, Rigby E. Federal food policy and childhood obesity: A solution or part of the problem? Health Affairs 2010; 29: 411-418.

11. Leung CW, Villamor E. Is participation in food and income assistance programmes associated with obesity in California adults? Results from a state-wide survey. Public Health Nutr 2011; 14: 645-652.

12. Gibson D. Food stamp program participation is positively related to obesity in low income women. J Nutr 2003; 133: 2225-2231.

13. Townsend MS, Peerson J, Love B, Achterberg C, Murphy SP. Food insecurity is positively related to overweight in women. J Nutr 2001; 131: 1738-1745.

14. Carrillo-Larco RM, Miranda JJ, Bernabe-Ortiz A. Impact of Food Assistance Programs on Obesity in Mothers and Children: A Prospective Cohort Study in Peru. Am J Public Health 2016; 106: 1301-1307.

15. Chaparro MP, Bernabe-Ortiz A, Harrison GG. Association between food assistance program participation and overweight. Rev Saude Publica 2014; 48: 889-898.

16. Third Report of the National Cholesterol Education Program (NCEP) Expert Panel on Detection, Evaluation, and Treatment of High Blood Cholesterol in Adults (Adult Treatment Panel III) final report. Circulation 2002; 106: 3143-3421.

17. Fukuyama N, Homma K, Wakana N, et al. Validation of the Friedewald Equation for Evaluation of Plasma LDL-Cholesterol. J Clin Biochem Nutr 2008; 43: 1-5.

18. Friedewald WT, Levy RI, Fredrickson DS. Estimation of the concentration of low-density lipoprotein cholesterol in plasma, without use of the preparative ultracentrifuge. Clin Chem 1972; 18: 499-502.

19. Chobanian AV, Bakris GL, Black HR, et al. The Seventh Report of the Joint National Committee on Prevention, Detection, Evaluation, and Treatment of High Blood Pressure: The JNC 7 report. JAMA 2003; 289: 2560-2572.

20. American Diabetes Association. 2. Classification and Diagnosis of Diabetes. Diabetes Care 2017; 40: S11-S24.

21. Barros AJ, Hirakata VN. Alternatives for logistic regression in cross-sectional studies: an empirical comparison of models that directly estimate the prevalence ratio. BMC Med Res Methodol 2003; 3: 21.

22. Leung CW, Willett WC, Ding EL. Low-income Supplemental Nutrition Assistance Program participation is related to adiposity and metabolic risk factors. Am J Clin Nutr 2012;
95: $17-24$.

23. Fernald LC, Gertler PJ, Hou X. Cash component of conditional cash transfer program is associated with higher body mass index and blood pressure in adults. J Nutr 2008; 138: 22502257.

24. Uauy $R$, Kain J. The epidemiological transition: need to incorporate obesity prevention into nutrition programmes. Public Health Nutr 2002; 5: 223-229.

25. Leung CW, Ding EL, Catalano PJ, Villamor E, Rimm EB, Willett WC. Dietary intake and dietary quality of low-income adults in the Supplemental Nutrition Assistance Program. Am J Clin Nutr 2012; 96: 977-988.

26. Nguyen BT, Shuval K, Njike VY, Katz DL. The Supplemental Nutrition Assistance Program and dietary quality among US adults: findings from a nationally representative survey. Mayo Clin Proc 2014; 89: 1211-1219.

27. Parks JC, Smith AD, Alston JM. The Effects of the Food Stamp Program on Energy Balance and Obesity. California, US: Davis Department of Agricultural \& Resource Economics; 2011.

28. Rucker IL. Is there an association between Supplemental Nutrition Assistance Program participation and elevated body mass index in women? Atlanta, US: Georgia State University; 2015.

29. Webb AL, Schiff A, Currivan D, Villamor E. Food Stamp Program participation but not food insecurity is associated with higher adult BMI in Massachusetts residents living in low-income neighbourhoods. Public Health Nutr 2008; 11: 1248-1255.

30. Instituto Nacional de Salud. Guía: Formulación de la ración del programa del vaso de leche. Lima, Perú: Ministerio de Salud, 2010

31. World Health Organization. Centro de Prensa: Alimentación Sana. Geneva, Switzerland: WHO; 2017 [updated 2017; cited 2017 November 8]; Available from: http://www.who. int/mediacentre/factsheets/fs394/es/

32. Blondet C, Montero C. Hoy: Menú Popular / Comedores de Lima. Lima: Instituto de Estudios Peruanos/UNICEF; 1995.

33. Diaz-Garces F, Vargas-Matos I, Bernabé-Ortiz A, DiezCanseco F, Trujillo A, Miranda JJ. Factors associated with consumption of fruits and vegetables among Community Kitchens customers in Lima, Peru. Prev Med Rep 2016; 4: 469-473.

34. Moore TJ, Alsabeeh N, Apovian CM, et al. Weight, blood pressure, and dietary benefits after 12 months of a Web-based Nutrition Education Program (DASH for health): longitudinal observational study. J Med Internet Res 2008; 10: e52. 